\title{
Análisis de mejora en la productividad del proceso de fabricación de rosquitas empleando un prototipo de formado de masa
}

\author{
Analysis of improvement in the manufacturing process of the rolls \\ production using a formed dough modeling
}

Álvaro Humberto Velásquez Dávila

Proavel, Servicio Nacional de Adiestramiento Industrial (SENATI)

\section{RESUMEN}

Objetivos: Implementación de mejoras en la productividad del proceso de fabricación de rosquitas mediante el uso de un prototipo de máquina electromecánica de formado de tiras trenzadas para producir rosquitas. Métodos: En el proceso de fabricación del prototipo los diferentes componentes, como el largo y grosor del sin fin, la potencia de los moto reductores, la velocidad rotacional del tornillo y diferentes variables de operación, como temperatura de extrusión, humedad y temperatura inicial de la masas de rosquita, se modificaron a fin de encontrar las condiciones óptimas de operación para que la rosquita sea de excelente calidad y no varie sus condiciones de sabor, forma y estructura reconocidas en el mercado. Resultados: Se diseñó un prototipo con un mecanismo de extrusión compuesto por un tornillo sin fin situado en una carcasa. En un extremo de dicha carcasa se ubica una tolva de alimentación de la masa de rosquita y por el otro se extruiría tiras por boquillas que al girarlas darían el trenzado de tiras requerido para el formado de la masa de rosquita. Se llevaron a cabo pruebas variando la humedad de la masa en 37,30 y $26 \%$, variando la temperatura de la masa y las revoluciones del tornillo $\sin$ fin. Conclusiones: Luego de las pruebas de humedad, temperatura y calidad, el mejor producto se obtuvo al utilizar una velocidad rotacional de $56 \mathrm{rpm}, 26 \%$ de humedad y utilizando masa a $25^{\circ}$ de temperatura. Asimismo, en términos económicos se ha logrado incrementar la productividad del negocio de rosquitas. Los costos de producción se han reducido en un $21 \%$ y el proyecto arroja un VAN positivo de S/. 7M.

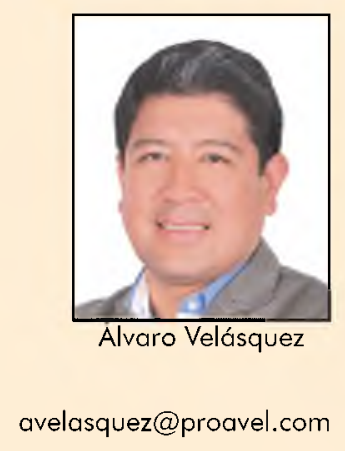

Historial del artículo: Recibido: 25 de noviembre de 2014 Aprobado: 10 de diciembre de 2014 Disponible en línea: 30 de diciembre de 2014

Palabras clave: Productividad, fabricación rosquitas, prototipo.

1 Economista, Master of Business Administration (MBA) de la Pontificia Universidad Católica del Perú, Gerente General de Procesadora de Alimentos Velásquez S.A.C. 


\section{ABSTRACT}

Objectives: Implementation of advances in the manufacturing process of rolls production by using a formed-braidedband electromechanical machine prototype. Methods: The process of manufacturing this prototype has been conducted to find the proper operating conditions. It has been modified different components such as the length and thickness of the endless, the force of reduced motors, the gyrator speed of the screw and different operating factors such as the extrusion temperature, humidity and initial temperature of the dough-making rolls in order to find the optimal operating parameters. As a result of these modifications, this type of product would have an excellent quality maintaining its recognized flavor, form and structure in the market. Results: It was designed an exemplar with a mechanism of extrusion consisting of a screw without end located in a housing box. In one side of its casing is a funnel of dough rolls and on the other end the squeezing bands for cavities that rotate them, which would give braiding bands required for forming the dough rolls. It has been carried out tests varying the amount of the humidity in $37 \%, 30 \%$ and $26 \%$; the temperature of the dough, and the speed of the screw. Conclusiones: After early tests showed of moisture, temperature and quality, the best product was obtained by using a rotated speed of $56 \mathrm{rpm}, 26$ $\%$ of humidity and used dough of $25^{\circ}$ of temperature. Additionally, in economic terms, the productivity of rolls has increased and the costs of production has been reduced in $21 \%$ providing a positive VAN of $\mathrm{S} / .7 \mathrm{M}$.

Keywords: Productivity, manufacturing rolls, prototype.

\section{INTRODUCCIÓN}

Para las empresas, la innovación es considerada una fuente de competitividad. Innovar en procesos productivos genera capacidades que permitan a las empresas adecuarse y competir en entornos de negocio muy agresivos. Para las pequeñas empresas es un reto aún mayor pues de ello depende en muchos casos su supervivencia. Según diversos autores se ha demostrado la relación directa que existe entre innovar y el crecimiento de las empresas, y por ende el crecimiento del PBI del país; algunos autores inclusive sostienen que la innovación es la piedra angular de un concepto más elaborado denominado desarrollo económico (1). En todo caso, el camino para las empresas se encuentra trazado, la innovación es el camino para la generación de valor de las empresas (2).

Sin embargo, nuestro país se encuentra en el puesto 75 a nivel mundial y 7 a nivel Latinoamérica y El Caribe en innovación. Se apreciatambién que no existe una correlación directa entre emprendimiento e innovación aun cuando el país es considerado una de los más emprendedores del mundo. Ello se debe a que el clima para la innovación no es favorable (3). Algunos autores sostienen que la innovación es una falla de mercado (4), se afirma que el mercado fracasa en generar incentivos para la innovación por lo que se debe de fomentar una política industrial desde el estado para lograr esos incentivos. Programas como FINCyT apuntan a corregir esa falla y a identificar proyectos innovadores de alto impacto en las empresas y por ende para el país.

PROAVEL es una pequeña empresa industrial dedicada a la fabricación y comercialización de productos de panadería, líder en los mercados de la región Junín con su marca Lalos. Uno de sus productos, la rosquita Lalos, requería de gran cantidad de mano de obra para su fabricación en la etapa del formado de rosquitas. La empresa buscó mejorar la productividad y la estandarización de la rosquita con maquinaría que no encontró en el mercado peruano. Por ello, la empresa PROAVEL asociada con el Servicio Nacional de Adiestramiento Industrial (SENATI), con el financiamiento del Programa de Ciencia y Tecnología (FINCyT) desarrollaron una máquina electromecánica para mejorar la productividad del formado del producto. 
En el caso de PROAVEL se requería pensar en un cambio en el proceso productivo de la rosquita. Hacerlo de una manera más eficiente implicaba automatizar el proceso de formado de rosquita (tiras trenzadas unidas por sus extremos) pues este requería mucha mano de obra además se requería corregir las deficiencias en la estandarización del producto básicamente tamaño, color y sabor del producto.

La ROSQUITA, es un producto de creación peruana. Consiste en unir dos tiras, trenzarlas, unirlas en círculo para formar el producto. En el mercado local no existe una máquina que automatice el proceso de formado de roscas.

Para la puesta en marcha del proyecto se pensó en la fabricación de un prototipo de extrusión. El proceso de extrusión es un procedimiento cada vez más popular en el procesamiento de harinas. El término general de "extrusión" designa la extracción de una sustancia mediante presión a través de una boquilla. Los extrusores están compuestos por uno o varios tornillos sin fin situados en una estrecha carcasa. Por un extremo se introduce la materia prima, por el otro, se extrae el producto a presión a través de boquillas. Mientras tanto, en algunos casos, la masa que se va a extrudir se procesa térmicamente mediante la calefacción de la carcasa y mecánicamente a través del molde, boquillas y del número de revoluciones de los tornillos sin fin.

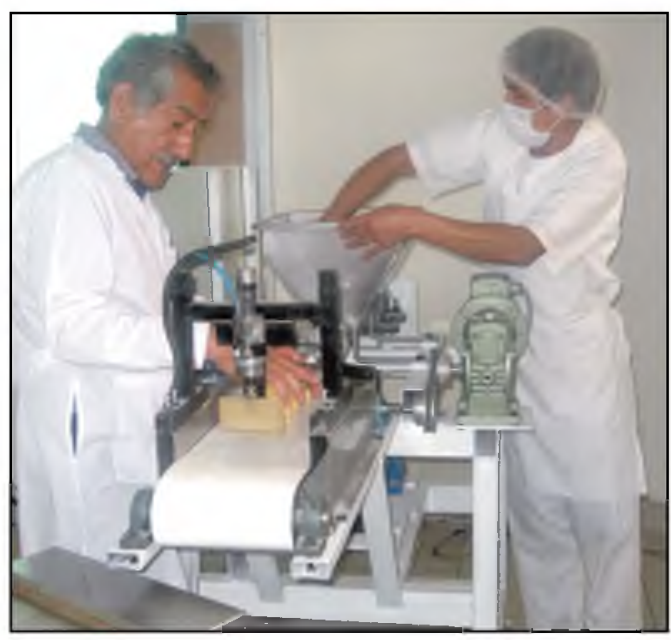

Figura $\mathrm{N}^{0}$ 1: Prototipo de máquina electromecánica para el formado de rosquita
En nuestro caso, la masa de rosquita pasa al tornillo sin fin, donde los ingredientes se someten a la influencia de temperatura, efecto mecánico y tiempo, tal y como ocurre a menor escala en el trabajo de mesa. Sin embargo, la temperatura de masa y la humedad cambian por efecto del extrusor. Al girar los tornillos sin fin, la masa se apelmaza y se calienta cambiando sus características fisicoquímicas pudiendo producir rosquitas de diferentes textura y calidad que las aceptadas en el mercado. No obstante, la calidad del producto no solo depende de los componentes de masa y resultados de extrusión, sino sobre todo de la destreza del operario de la máquina. En el estudio se muestran los resultados de las pruebas hechas a diferentes temperaturas de masa y humedad para lograr una rosquita de excelente calidad.

Es interesante anotar que esta nueva tecnología puede permitir el desarrollo de nuevos negocios para la empresa. Se pueden crear formas interesantes de presentación de las rosquitas que resultan muy atractivas para el consumidor. Asimismo, ofrecer alimentos más nutritivos, más limpios, estandarizados y sabrosos posibles.

El objetivo de esta investigación fue evaluar la producción de rosquitas utilizando el prototipo desarrollado con el financiamiento del FINCyT y posteriormente, evaluar los resultados relacionados con la creación de valor para la empresa.

\section{MATERIAL Y MÉTODOS}

\section{Materiales y condiciones de extrusión}

Luego de un proceso muy largo de prueba y error de partes y piezas, se desarrolló una máquina para el labrado de las trenzas de rosquitas con las siguientes dimensiones; largo de $1620 \mathrm{~mm}$, ancho de $700 \mathrm{~mm}$, altura de $1380 \mathrm{~mm}$ y peso de $170 \mathrm{~kg}$.

La máquina está conformada por tres motores con reductores de velocidad y guillotina: 
1. Motoreductor de $1 \mathrm{HP}$ de 1600 RPM con una relación 1:30 que nos da 56 RPM final. A dicha velocidad gira el tornillo sin fin.

2. Motoreductor de 0,5 HP con una relación de 1:60 que mueve la faja transportadora.

3. Motoreductor de 0.5 HP que hace girar la boquilla para formar las trenzas.

4. Selenoide de 220 VDC (Voltaje Directa Corrient) de 1,2 AMP de 40 Wats, que hace mover la guillotina de corte.

El mecanismo extrusor está conformado por un tolva de recepción de masa de 5 $\mathrm{kg}$, un tornillo sin fin de dos pulgadas $y$ media y un largo de $45 \mathrm{~cm}$. El equipo es totalmente desmontable para su limpieza y mantenimiento por su naturaleza alimentaria. El equipo se ha elaborado de acero inoxidable y PVC permitidos en la industria.

Tabla $N^{\circ}$ 1: Inversion final del prototipo en Nuevos Soles.

\begin{tabular}{lrrrr}
\hline Entidad & Monetario & \multicolumn{1}{c}{$\begin{array}{c}\text { No } \\
\text { monetario }\end{array}$} & Total & $\%$ \\
\hline $\begin{array}{l}\text { Innovate } \\
\text { Perú }\end{array}$ & 79,900 & & 79,900 & 72,36 \\
$\begin{array}{l}\text { Fidecom } \\
\text { Proavel }\end{array}$ & 10,926 & 10,000 & 20,926 & 18,95 \\
$\begin{array}{l}\text { S.A.C. } \\
\text { Senati }\end{array}$ & & 9,600 & 9,600 & 8,95 \\
Total & 90,826 & 19,600 & 110,426 & 100,00 \\
\hline
\end{tabular}

Tabla $N^{\circ}$ 2: Pruebas de humedad y temperatura de masa.

Humedad. Cantidad de agua en masa

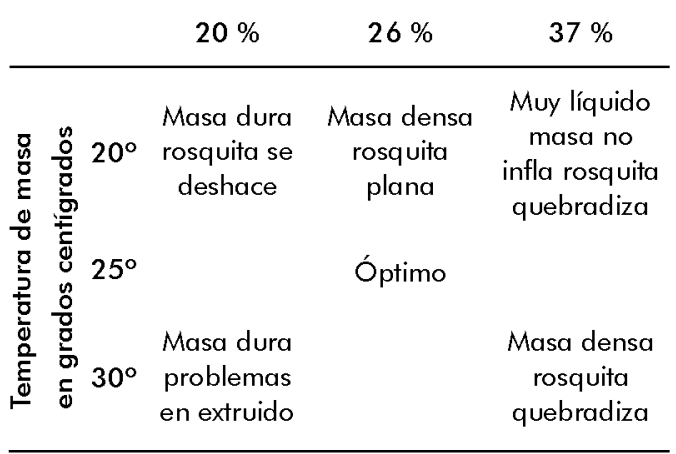

Tabla N ${ }^{\circ}$ 3: Encuesta de evaluacion sensorial del producto.

\begin{tabular}{ll}
\hline 5 & Gusta Mucho \\
4 & Gusta Moderadamente \\
3 & Gusta Poco \\
2 & Me es indiferente \\
1 & Me disgusta \\
\hline
\end{tabular}

El sinfín se encuentra ubicado en una cámara y fijado en dos muescas del eje impulsor. A su vez el sin fin se fija por la parte posterior con un tornillo de sujeción. La cámara de alimentación de la boquilla tiene dos partes que se unen con una brida de 6 pernos que se ajustan a la parte anterior de la cámara principal.

Se alimenta la tolva con la masa y se espera que empiece a salir las tiras, para luego girar las boquillas y lograr el trenzado.

Asimismo, consta de un tablero de control con tres variadores de velocidad que nos permite graduar la velocidad de la faja transportadora, la cantidad de vueltas de la boquilla y la distancia de corte de las trenzas.

La inversión final del proyecto se detalla en la tabla $N^{\circ} 1$.

Se obtuvieron rosquitas elaborados a partir de diferentes condiciones de humedad inicial de la masa (37\%, $30 \%, 26 \%)$, temperatura de masa $\left(20^{\circ}, 25^{\circ}\right.$ y $\left.30^{\circ}\right)$ a una velocidad de tornillo extrusor de 56 RPM. Así se encontró que las condiciones óptimas para trabajar la masa en el extrusor eran de $26 \%$ de humedad, $25^{\circ}$ de temperatura para lograr tiras consistentes y uniformes que permitieran rosquitas de excelente calidad, tabla $\mathrm{N}^{\circ} 2$.

\section{Evaluación sensorial}

La evaluación sensorial tuvo como finalidad confirmar que las rosquitas tuvieran el mismo sabor y calidad que aquellas que son labradas a mano y reconocidas en el mercado. Esta evaluación se llevó a cabo por medio de una escala hedónica 
estructurada (del 1 al 5) donde se calificó el nivel de agrado de cada producto de acuerdo a la tabla $\mathrm{N}^{\circ} 3$.

Se tomó una encuesta con una muestra de 30 observaciones para conocer el nivel de agrado.

\section{RESULTADOS}

Es interesante notar que la media se encuentra en "Gusta Moderadamente" lo cual indica aprecio del consumidor por el producto, el valor más frecuente se encuentra en "Gusta Mucho" y la posición central de los consumidores se encuentra en "Gusta moderadamente", tabla N ${ }^{\circ} 4$

Con esta información concluimos que la máquina ofrece un producto bueno $y$ aceptable por el mercado.

\section{Resultados técnicos del Prototipo}

Los resultados han sido exitosos y se cumplieron los objetivos trazados en la formulación del proyecto.

Se logró estandarizar las rosquitas con las siguientes características:

- Peso: $13 \mathrm{~g}$.

- Tamaño: $8 \mathrm{~cm}$ de diámetro y forma de argolla.

El proyecto planteaba obtener como mínimo una capacidad de procesamiento de $4 \mathrm{~kg}$ masa por hora en la etapa de formado (291 unidades por hora). Se ha logrado una capacidad de procesamiento de 11,7

Tabla N ${ }^{\circ}$ 4: Resultados de encuesta

\begin{tabular}{lc}
\hline & \\
\hline Media & 4,033 \\
Error típico & 0,206 \\
Mediana & 4,000 \\
Moda & 5,000
\end{tabular}

kg masa por hora (900 unidades por hora).

El proyecto planeaba reducir el tiempo de formado de masa en un 30 \%. Las horas hombre utilizadas sin máquina para un batch de producción (1 Batch hace referencia a 1 carro de horneado; 1 Batch de Rosquita equivale a 1386 rosquitas) alcanzaban las 6,6 horas hombre. Con el prototipo se reduce a 1,54 horas hombre. Una reducción del $76 \%$.

Se redujeron las mermas por cada batch en más de un $20 \%$.

Se redujo el costo del batch de producción en un $21 \%$.

Se ha capacitada a un personal en la utilización de la máquina y a otro en el mantenimiento de la misma.

Estos resultados permiten afirmar que los objetivos planteados por las entidades participantes del proyecto se han cumplido.

\section{Analisis de generacion de valor del nuevo proceso productivo}

El principal impacto de la incorporación de la máquina ha sido disminuir el costo de la mano de obra. Esta pasa de 6 a 1,54 Horas hombre $(\mathrm{H} / \mathrm{H})$ para la producción de 1 batch de rosquitas. Esa reducción del costo compensa ampliamente los costos que ahora se presentarán con la utilización de máquina: Depreciación, Mantenimiento y Seguro, tabla $N^{\circ} 5$.

Es interesante destacar dos aspectos. El primero que el costo de formado se reduce

Tabla $N^{\circ}$ 5: Costo del formado de masa.

\begin{tabular}{|c|c|c|}
\hline \multicolumn{3}{|c|}{ Soles por Batch de producción } \\
\hline & Sin máquina & Con máquina \\
\hline Costo de mano de obra & 32,20 & \\
\hline Costo de mano de obra & & 8,27 \\
\hline Depreciación de equipo & & 5,69 \\
\hline Mantenimiento & & 3,85 \\
\hline Seguro equipo & & 1,42 \\
\hline Total & 32,20 & 19,22 \\
\hline
\end{tabular}


Tabla $N^{\circ}$ 6: Participación de los diferentes componentes de costo.

\begin{tabular}{lrr}
\hline & & \\
\hline \multicolumn{1}{c}{ ltems de costo } & Sin máquina & Con máquina \\
\hline Insumos & $33 \%$ & $37 \%$ \\
Formado & $24 \%$ & $16 \%$ \\
Horneado & $2 \%$ & $2 \%$ \\
Empacado & $12 \%$ & $14 \%$ \\
Pérdida industrial & $2 \%$ & $1 \%$ \\
Gastos generales/ & & \\
financieros/ & $27 \%$ & $30 \%$ \\
comercialización & & \\
Total & 32,20 & $100 \%$ \\
\hline
\end{tabular}

Tabla N ${ }^{\circ}$ 7: Cálculo de rentabilidad (paquete $\mathrm{x}$ 8).

\begin{tabular}{lrc}
\hline & & \\
\hline & $\begin{array}{c}\text { Nuevos Soles } \\
\text { Sin máquina }\end{array}$ & Con máquina \\
\hline Margen & $25 \%$ & $34 \%$ \\
\hline
\end{tabular}

en $40 \%$ utilizando la máquina. Pasa de S/. 32,20 a S/. 19,22 por batch de producción. El segundo es que los costos de la máquina se encuentran asociados a la cantidad de producción. Si esta producción se incrementa, los costos de producir con la máquina son menores. Así, la producción actual de la empresa sin máquina es de 1 batch diario y se espera duplicar la producción a 2 batch el 2015. Con este incremento de producción los costos asociados a la máquina se reducirían a $\mathrm{S} /$. 13,74 y reflejarían una diminución de costo en $57 \%$.

Cabe señalar que el costo del formado es sólo una parte del costo total. La utilización de la máquina reduce los costos totales en un 11,2 \% monto importantísimo para la mejora del margen en este producto. Un aspecto importante es que la máquina permite reducir la merma industrial en un $69 \%$ aunque si bien el monto de ahorro es pequeño es un aspecto importante en los indicadores de eficiencia del uso del equipo, tabla $\mathrm{N}^{\circ} 6$.

La rentabilidad final del producto rosquita se detalla en la tabla $N^{\circ} 7$.

Para el cálculo del Valor Actual Neto del
Tabla $N^{\circ}$ 8: Analisis de generación de valor.

\begin{tabular}{lr}
\hline & \multicolumn{1}{c}{2015} \\
\hline Unidades vendidas & 108108,00 \\
Margen & $41 \%$ \\
Utilidad & 44225 \\
I renta & 13267 \\
Utilidad neta & 30957 \\
Depreciación & -11043 \\
Flujo caja & 19,915 \\
Flujo de caja perpetuo & 117,145 \\
& \\
Menos inversión total & 110,426 \\
Van del proyecto & 6,719 \\
\hline
\end{tabular}

Negocio se ha supuesto, en un escenario extremadamente conservador, que las ventas se mantendrán a un ritmo de 2 batch diarios. No se ha supuesto crecimiento de ventas aunque ello es muy probable toda vez que el producto es demandado y la empresa ha logrado ingresar a nuevos puntos de ventas como Metro y Plaza Vea en la ciudad de Huánuco.

Asimismo, la máquina permite hacer más eficiente la producción de algunos productos de la empresa como tiras de turrón y crisinos. Asimismo, permite el desarrollo de nuevos productos y atender mercados aún no explorados por la empresa.

El resultado es un flujo a valor presente de S/. 117145 que restándole el costo de la inversión de S/. 110426 nos da como resultado un VAN positivo de $\$$ /. 6719 para la empresa. Un proyecto que ha permitido la mejora de la eficiencia y la rentabilidad de la empresa.

\section{DISCUSIÓN}

Las conclusiones a las cuales se han arribado a la culminación del proyecto son:

1. El prototipo cumple los objetivos trazados al inicio del proyecto. Ha mejorado la productividad de la empresa disminuyendo las horas hombre requeridas en el proceso de labrado. Ello ha tenido un impacto 
significativo en el costo generando un ahorro de $21 \%$ y un incremento en el valor de la empresa.

2. La capacidad de procesamiento se ha ampliado en casi 5 veces. La máquina puede ser usada para otros productos y alcanzar nuevos mercados.

3. Se ha logrado estandarizar el producto rosquita y disminuir las mermas de producción en un $20 \%$.

4. Se ha logrado capacitar a dos empleados. Uno en el manejo de máquina y otro en el mantenimiento de la misma. Son ahora trabajadores con mayores competencias y productividad mayor.

\section{Agradecimientos:}

A FINCyT por el financiamiento del proyecto "Prototipo de máquina electromecánica para el formado de tiras de masa de rosquitas".

\section{REFERENCIAS \\ BIBLIOGRÁFICAS}

1. Repositorio institucional: Palestra, Portal de Asuntos Público [Internet]. Lima: Pontificia Universidad Católica del Perú; 2013 Septiembre [Citado el 19 de enero de 2014]. Desarrollo e Innovación [1 pantalla]. Disponible en: http://repositorio.pucp.edu.pe/index/ handle/123456789/11798

2. Martin de Holan P Crecimiento Rentable e Intrapreneurship. Strategia. 2011; (21): 65-66.

3. Falcón D. Ecosistema de la innovación en Perú. Diario Gestión. 3 de mayo de 2012; Blogs. Disponible en: http://blogs.gestion.pe/ innovaciondisrupcion/2012/05/ ecosistema-de-la-innovacion-en.html

4. Callejon $M$. ¿Qué política industrial y tecnológica precisan las pequeñas y medianas empresas? Economía Industrial. 2010; (375): 139-150.
5. Industria Alimenticia [Internet]. Troy: Ivan Rioja-Scott; 2010 Abril [Citado el 19 de enero de 2014]. Fundamentos de la Extrusión [1 pantalla]. Disponible en: http://www.industriaalimenticia. com/articles/83076-fundamentos-dela-extrusion

6. Industria Alimenticia [Internet]. Troy: Ivan Rioja-Scott; 2008Febrero [Citado el 19 de enero de 2014]. Comercializando la Extrusión [1 pantalla]. Disponible en: http://www.industriaalimenticia.com/ articles/83030-comercializando-laextrusion. 